\title{
木本植物水力学结构之导管长度研究进展
}

\author{
程向芬 ${ }^{1}$ 马 晋 ${ }^{1}$ 赵 涵 ${ }^{1}$ 姜在民 ${ }^{2}$ 蔡 靖 $1,3^{*}$
}

${ }^{1}$ 西北农林科技大学林学院, 陕西杨凌 712100; ${ }^{2}$ 西北农林科技大学生命学院, 陕西杨凌 $712100 ;{ }^{3}$ 陕西秦岭森林生态系统国家野外科学观测研究站, 陕西杨凌 712100

摘 要 导管作为多数被子植物木质部水分运输的主要通道, 了解其结构及功能对研究被子植物水力学特性及植物对环境 的适应性有着重要的作用。导管长度作为导管解剖特征之一, 对水分运输的安全性及有效性有着重要的影响。该文概述了导 管长度测量及计算的方法, 导管长度在种内及种间的分布, 导管长度与导管直径的关系, 导管长度与导水率的关系及导管长 度对建立栓塞脆弱曲线的影响, 并对未来导管长度的研究工作重点提出了建议: 1)改进灌注物质, 使灌注更加充分且更利于 观察、提高计算精度、发展活体动态测量技术; 2)建立导管在植物不同器官及整体的分布网络以及不同生活型、不同地区的 导管长度数据库; 3)对导管直径在导管方向的变化, 导管长度与其他导管特性之间的关系进行研究; 4)光学测量建立栓塞脆弱 曲线技术的兴起, 可为解决离心机法建立栓塞脆弱曲线的真实与准确与否的争议提供新的方向。更深入地了解导管长度在植 物水力功能中担负的角色, 可以为耐旱、抗旱品种选育提供理论基础。

关键词 染料注入法; 空气注入法; 栓塞脆弱曲线; 水力学特性

程向芬, 马晋, 赵涵, 姜在民, 蔡靖 (2018). 木本植物水力学结构之导管长度研究进展. 植物生态学报, 42, 609-618. DOI: 10.17521/cjpe.2017.0293

\section{Vessel length as a key hydraulic structure in woody plants: A review}

\author{
CHENG Xiang-Fen ${ }^{1}$, MA Jin ${ }^{1}$, ZHAO Han ${ }^{1}$, JIANG Zai-Min ${ }^{2}$, and CAI Jing ${ }^{1,3^{*}}$ \\ ${ }^{1}$ College of Forestry, Northwest A\&F University, Yangling, Shaanxi 712100, China; ${ }^{2}$ College of Life Sciences, Northwest A\&F University, Yangling, Shaanxi \\ 712100, China; and ${ }^{3}$ Qinling National Forest Ecosystem Research Station, Yangling, Shaanxi 712100, China
}

\begin{abstract}
Vessels are the conduit for water transport in xylem of most angiosperms. Our knowledge on their structure remains crucial in understanding the hydraulic properties of angiosperms and their adaptability to the changing environment. Vessel length is one of the major anatomical features that play a fundamental role in determining the trade-offs between safety and efficiency of long-distance water transport in xylem. This article provides a review of scholarly research on the structure and function of plant vessels, as well as the corresponding methods, measurements, scientific challenges on the influence on vulnerability curve and the relationships with other features of vessel length, as well as our knowledge gaps. Future efforts should be placed on: 1) improvement of the materials for penetration for their completion, easy-to-use, measurement accuracy, and new technology; 2) sound research on vessel network of different organs, as well as a database on vessels of different functional groups and climate zones; 3 ) the change in vessel diameter and other structural features with length; 4) optical measurements of xylem vulnerability for additional evidences on the roles of artificial open vessels. Advancing our knowledge on the role of vessel length in hydraulic function of plants will provide us with a theoretical foundation for improvement of drought-tolerant and drought-resistant species.
\end{abstract}

Key words paint injection; air injection; vulnerability curves; hydraulic characteristics

Cheng XF, Ma J, Zhao H, Jiang ZM, Cai J (2018). Vessel length as a key hydraulic structure in woody plants: A review. Chinese Journal of Plant Ecology, 42, 609-618. DOI: 10.17521/cjpe.2017.0293

导管作为木本植物木质部中运输水分与无机盐 的重要管道结构, 普遍存在于大多数被子植物中。 它们是由许多长管状的细胞以顶端对顶端的方式连 接而成的管状结构。组成导管的每一个细胞称为导 管分子, 单个导管由成百上千个死亡的导管分子组
成，导管分子在发育初期是活的细胞，在成熟过程 中, 细胞侧壁木质化并产生次生加厚, 同时, 细胞 内的液泡膜破裂, 释放出水解酶, 使上下相连的两 个导管分子之间的端壁溶解消失形成穿孔板, 以便 于水分在导管分子间的长距离运输, 原生质体解体 
而成为死细胞, 整个导管成为一个长管状结构, 长 度可达0.001-10 m (Cai \& Tyree, 2014), 是导管直径 的100-1 000倍(Pan et al., 2015)。根据侧壁纹孔的排 列方式, 人们将导管分为环纹、螺纹、梯纹、网纹 和孔纹等不同类型。导管分子的进化过程与维管植 物的进化是相联系的, 穿孔板与纹孔类型在进化过 程中都发生了各自的变化, 导管直径变大、长度缩 短、连接度增高被普遍认为是导管的进化趋势(李红 芳等, 2005)。

木质部导管特征是决定植物水力结构和功能的 关键因素, 其直径、长度、内径跨度、壁厚度、连 接度等特征都对植物的水力结构与功能有着重要的 影响。植物的水分运输, 不是由一条导管从底部通 到顶端的，而是分段经过许多条导管曲折连贯向上 运行的。水流既可以通过导管细胞腔及穿孔上升, 又可以通过侧壁上的纹孔横向运输。导管中的水分 在运输过程中, 受张力影响处于亚稳定状态, 在地 上部分与地下部分的压力差下, 水分以水柱的形式 由木质部根的导管向上运输到茎的导管(李荣等, 2015), 最终到达叶片等器官。干旱、冻融交替等损 伤, 会使得空气、灰尘等经由纹孔膜进入充水管道, 即形成空穴化, 并进一步形成栓塞, 即整个导管为 气体占据, 使植物水力学功能受到影响(Tyree \& Dixon, 1986; Sperry \& Tyree, 1988)。栓塞会造成植物 水力功能障碍, 影响水分运输甚至是植物的正常生 长。木质部的进化使得水分运输的效率更高, 但更容 易受空穴化的影响, 植物需要既安全又有效的运输 形式, 安全性与有效性之间的差异可以用来反映物 种在生态位及进化上的差异(Gleason et al., 2016)。 即使未发生栓塞, 导管的输导功能也并非是永久保 持的, 新的导管形成后, 老的导管会因周围薄壁细 胞的侵入形成侵填体而相继失去输导水分的能力。

目前研究多集中在导管内径的测量与功能上, 较少关注导管长度, 对导管长度本身及其在植物水 力结构及功能中的作用缺乏系统的了解(Jacobsen et al., 2012)。究其原因是导管特殊的几何结构特点, 无法在显微镜下直接观察测量其长度(Cai \& Tyree, 2014), 需借助其他手段及方法等进行测量及计算。 而导管长度测量的复杂性, 也是导管长度研究目前 被严重忽略的重要原因(Ewers \& Fisher, 1989a; Comstock \& Sperry, 2000)。本文将从导管长度测量 与计算, 导管长度在水力功能中的作用, 导管长度
研究中的问题以及未来研究方向等方面展开论述。

\section{1 导管长度测量方法}

\section{1 拍照法}

测量导管长度最直接的方法为显微拍照法在光学显微镜下将一根导管从头至尾拍下, 但由于 导管特殊的几何形状, 使得导管长度的测量需拍摄 成百上千张照片进行拼凑, 工作量大且精度较低, 因此不推荐使用该方法(Ewers \& Fisher, 1989a; Comstock \& Sperry, 2000; Tyree \& Zimmermann, 2002)。

\section{2 注射法}

导管长度测量的间接方式是将有色物质注入人 为切开的导管使其形象化, 该物质选择的原则是: 被注入导管后, 能够在贯穿导管的基础上不发生堵 塞, 也无法穿过纹孔膜扩散到相邻导管(Cai \& Tyree, 2014)。灌注前一般用冲洗法 $\left(0.1 \mathrm{~mol} \cdot \mathrm{L}^{-1} \mathrm{KCl}, 0.12\right.$ $\mathrm{MPa}, 30 \mathrm{~min})$ 将导管中的残余气泡除去, 因为在灌 注过程中, 灌注物质会在导管中推着气泡向前, 直 至将气泡压缩至压强与液压相等, 灌注物质无法继 续向前, 导致灌注不充分。灌注完成后室温下静置 干燥, 然后在距离灌注端不同位置处取样切片, 在 显微镜下拍照, 观察被灌注导管的数量(截面导管 数量或单位面积导管数量), 能够观察到导管填充 数量的变化。

早期多采用油基漆作为灌注物质, 以一定的压 力沿导管运输方向注入枝条, 在导管中, 油会取代 水到达导管末端, 水油表面张力较大, 在灌注压力 较小的前提下油不会透过纹孔膜进入邻近的导管, 但该过程通常需要数天。灌注完成后, 在近灌注端, 有些导管完全灌注, 有些则是完全不含灌注物, 使 计数不充分, 在干燥过程中, 多数导管中的油基漆 收缩, 体积变小, 无法充满整个导管, 使得计数难 度增加(Zimmermann \& Jeje, 1981)。之后, 很多人用 100 倍稀释后的水基乳胶漆代替了油基漆, 但该方 法仍存在较多缺陷。乳胶漆本身颜色较浅, 且其中 的非晶体颗粒容易集结、吸附在颜料离子周围, 形 成大的团状聚合体, 无法通过穿孔, 还未到达导管 末端便发生堵塞, 这一点在小直径导管上尤为明显, 即使在0.1 MPa下灌注一周颜料仍无法充满整个管 腔, 在观察导管数量时造成误差, 所测平均导管长 度偏小(Sperry et al., 2005)。也有用热蜡作为灌注物 质的, 与水基乳胶漆类似, 也存在灌注不充分的问 
题(Zimmermann \& Jeje, 1981)。如今多使用即时配置 的硅胶溶液 (10 g RTV141A + 1 g RTV141B) 与荧光 剂 $(0.5 \mathrm{~mL}, 1 \%$ 苂光增白剂/氯仿 $(\mathrm{m} / \mathrm{m})$ ) 混合(André, 1998; Sperry et al., 2005; Hacke et al., 2007)。苂光剂 不溶于水故不会通过纹孔扩散到相邻导管, 引起测 量误差。灌注完成后需室温静置干燥 3 天左右, 时间 大大缩短(Cai \& Tyree, 2014)(图1)。和其他方法相比, 混合硅胶解决了先前灌注物质存在的粒子结块、堵 塞导管、灌注不充分、流动性弱、干燥时间过长等 问题。

\section{3 空气注入法}

空气注入法是一种相对快速的导管长度动态测 量方法。是在一定的压力下向枝条注入空气, 并根 据收集气泡的速率与测试样本长度的关系来推算不 同长度导管的概率分布。在第一个气泡出现后, 测 量此时单位体积空气在单位时间内通过的距离(空 气流速), 此时导管长度为该样本的最大导管长度 $L_{\max }$ 。然后将枝条逐渐剪短, 重复操作。该方法的前 提为假设开口导管的数量与气体导度成正比, 并根 据枝条长度与空气流速间的关系来推算导管长度分 布。当被切开的新鲜茎段在低压 $(<100 \mathrm{kPa})$ 下注入气 体时, 气体只能通过被切开的导管, 因为导管壁上 的湿润纹孔膜可以阻止气流的通过, 使气流不能到 达相邻导管(Skene \& Balodis, 1968)。气体灌注压力 恒定时, 压力梯度与流量均随茎段长度的减小而增 加。茎段对空气的传导率 $(C)$ (单位时间内, 通过单位 横截面积的空气的体积)与开口导管长度无关, 而 是与茎段中导管的数量和导管直径大小成比例(Pan et al., 2015)。当枝条长度减小到一定程度时, 由于 没有新的导管被切开, 气体导度将不再增大。因此, 气体导度与导管数量有关。根据Hagen-Poiseuille方
程的预测，空气流速与开口导管直径的4次方有关， 且大直径导管对水分运输与空气流通的贡献更大 (Tyree \& Zimmermann, 2002)。因此空气注入法所得 应是水力功能加权后的导管长度分布, 受大直径导 管影响较大。且测量最大导管长度时也多用空气注 入法(Jacobsen et al., 2012)。

在用空气注入法进行导管长度测量时, 随着枝 条剪短, 不同枝条长度 $(x)$ 时分别测得一个相应的 $C$, 并且在茎段中导管随机分布的假设前提下, 可推算 出茎段的 $C$ 的减少量和茎段长度的变化量 $d x$ 成比例, 因为随着茎段的增长, 导管数量也在增加, 可推导 出公式(1):

$$
d C=C_{0}-C=C_{0}(-k d x) \text { 或 } d C / C_{0}=-k d x
$$

式中, $C_{0}$ 为茎段长度 $x$ 趋于 0 时的空气传导率; $k$ 为耗 散系数(消亡系数)。对于一个有限长度的茎段, 长为 $x$, 对于公式(1)积分可得:

$$
\begin{gathered}
\ln C-\ln C_{0}=-k x \text { 或 } \ln C=\ln C_{0}-k x \\
C=C_{0} \exp (-k x)
\end{gathered}
$$

公式(2)适用于不同长度茎段的 $C$ 分析。

对 $\ln C$ 和 $x$ 进行线性回归分析, 可以得到 $C_{0}$ 和 $k$ 。 设一个换算因子为 $D$, 若单位传导率的导管数量已 知, 则在进气端的导管数量为 $D C_{0}$ 。类似的, 可以用 引入相同的换算因子 $D$ 来得出 $L_{\max }$ :

$$
L_{\text {max }}=\ln \left(D C_{0}\right) / k
$$

用染料注入法测量导管长度时, 距离注射端不 同距离 $x$ 处的单位横截面积导管数量为 $N($ 横截面所 有被硅胶注射导管的数量/横截面的面积), 以 $x$ 为横 坐标, $\ln N$ 为纵坐标进行线性拟合, 斜率为 $\lambda_{v}$, 且满足:

$$
N=N_{0} \exp \left(\lambda_{\mathrm{v}} x\right)
$$

式中, $N_{0}$ 是近注射端处的单位横截面积导管数量 $(x$ 为 0.1 或 $0.2 \mathrm{~cm}$ ), $\lambda_{\mathrm{v}}$ 为拟合系数 (同公式 1 中的 $k$ )。
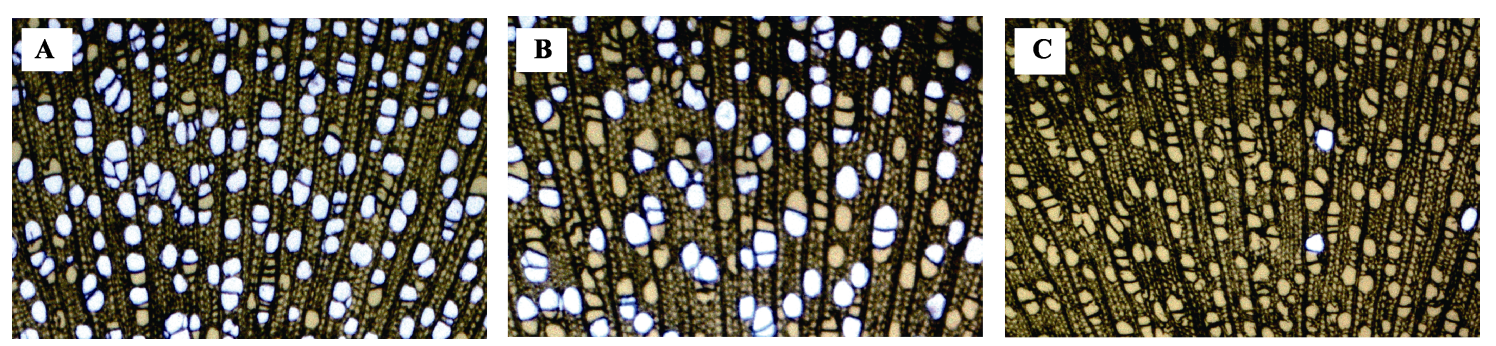

图1 硅胶注射法测定Populus tremuloides 导管长度。A、B、C依次为距离灌注端0.5、1.0和5.0 cm的苂光图片, 亮色部分为紫 外灯照射下的被硅胶灌注的导管(蔡靖摄)。

Fig. 1 The vessels of Populus tremuloides with injection of silicone rubber mixed with fluorescer. A, B, C are the observations at $0.5,1.0$ and $5.0 \mathrm{~cm}$ from the injection surface, respectively. The brightness indicates the rubber-filled vessels under UV light (Photographed by CAI Jing). 
无论是染料注入法利用测得的被灌注导管的数 量或是空气注入法用不同长度茎段下的气体传导率, 皆通过双差法来描绘导管长度分布(Tyree, 1993), 双差法的表达式(Zimmermann \& Jeje, 1981)如下:

$$
P_{x}=x\left(d^{2} C / d x^{2}\right) / C_{0}
$$

$P_{x}$ 为长度为 $x$ 的导管所占的比率, 将公式(2)二次 积分, 代入公式(5)中, 可得出连续型概率密度函数:

$$
P_{x}=x k^{2} \exp (-k x)
$$

Cohen等(2003)经过计算已经证明公式(6)是一 个Gamma概率分布函数, 它的形状因子等于 2 , 对 公式(6)求导数可得:

最高频率导管长度 $L_{0}=-1 / k$

平均导管长度 $\overline{L_{\mathrm{v}}}=-2 / k$

以上的计算方法目前被广泛使用, 但其结果仍 与实际值存在偏差, Cai 和 Tyree (2014) 在计算 Populus tremuloides 导管长度分布概率时, 对公式(2) 中的 $\ln C$ 和 $x$ 进行了二阶多项式的回归, 将拟合精度 提高了3倍左右, 且拟合结果也更符合杨树短导管 数量更多的情况。

\section{4 高分辨率断层扫描技术}

还有一种测量方法是应用高分辨率的X射线计 算机断层扫描技术(HRCT), 并配合分析软件来构 建包括导管直径、导管长度、导管连接等在内的木 质部整体网络结构。HRCT与医用CT技术原理类似, 可高强度、高分辨率地在植物组织的任何部分连续 进行切片拍摄, 且不会对内部组织造成损伤(Brodersen et al., 2010), 将拍摄的图片用自定义开发的分 析软件(Avizo)进行处理, 先建立初始的网络结构, 然后根据物种的解剖特性进行修改完善。建立的三 维图像能够观察一定区域的全部导管及周围组织的 完整结构, 并自动测量导管长度及直径等指标。由 于该技术测量长枝条所用时间较长, 且该显微摄影 技术需要先进的X射线发射源的显微断层摄影设备 支持, 对硬件要求较高, 因此目前较少得到应用。但 该技术能够无损观测植物组织内部, 其建立的三维 木质部导管网络是手动测量无法比拟的, 因此在植 物解剖及生理学上具有良好的应用前景(Brodersen et al., 2011)。

\section{5 不同测量方法的比较}

拍照法是最直接的导管长度测量方法, 能够较 为直观地观察到导管的微观形态, 但由于测量一根 导管所需的照片数目较大, 测量时需对照片进行拼
凑, 导致工作量大且精度低, 因此并不推荐使用。

染料注入法将导管形象化, 测量结果较为准确, 能够直观地观察导管长度的分布状况, 明显看到导 管长度与导管直径的关系, 但其测量周期长, 且最 早的染料注射法溶剂多为油基漆等凝固较快的液体, 注入导管内的染料粒子易发生凝聚现象, 堵塞导管, 容易出现灌注不充分的情况。后期开始使用加了苂 光剂的硅胶进行注射, 相较之前的注射剂具有凝固 较慢, 粒子不易成团等优点, 在低压下, 更能趋近 于导管终点, 所得结果更加准确, 也是目前被广泛 使用的测量方法。染料注入法也具有自身的缺陷, 由于需要在低压下将染料注入枝条内, 灌注完成后 需静置干燥, 耗时较长, 无论改进前的油基漆法还 是改进后的硅胶注射法, 普遍存在难以到达导管底 部的现象, 使测量结果存在一定误差。

空气注入法相对于染料注入法, 操作比较简单, 测量周期更短, 但普遍认为该方法所得导管长度并 非传统意义上的导管长度, 而是与植物水力学特性 相关的水力学长度(Venturas et al., 2016; Pan et al., 2015), 当测量的导管长度与水力特征有关时, 空气 注入法可能更适合。空气注入法不存在染料注入法 的灌注不充分问题, 在生长季后期, 导管中产生胼 胝体但尚未完全堵塞时, 染料注入法测量精度会大 大降低, 而空气注入法则不受影响(Pan et al., 2015)。Pan等(2015)用两种方法分别对环孔材(栓皮 栎Quercus variabilis和葡萄Vitis vinifera)、散孔材(元 宝枫Acer truncatum) 以及半环孔材(84K杨树Populus $a l b a \times$ Populus glandulosa) 的当年生枝条进行测量, 结果发现空气注入法所得 4 个种的平均导管长度普 遍大于硅胶注射法所得的平均导管长度, 且二者存 在显著性差异, 作者认为该结果可能是由于硅胶注 射法是通过硅胶形象化的导管长度来计算, 而空气 注入法则是基于测得的空气导度来推算的。但在 Jacobsen等(2012)的研究中, 不同测量方法所得的8 个树种的平均导管长度是一致的, 彼此之间并没有 显著差异。综上所述, 两种测量方法所得结果的差 异性及其原理还需要更多的研究来验证。

高分辨率断层扫描技术借鉴了医学上的计算机 断层扫描技术, 分辨率更高, 对植物材料进行连续 扫描, 具有精度高、直观化、视野范围广、活体测 量、可建立立体化导管网络等优点, 但由于所需仪 器及配套设施较为昂贵, 使用及维护不便, 目前难 
以被广泛应用于植物解剖结构的观测尤其是田间试 验上(Brodribb et al., 2017)。

\section{2 导管长度的分布}

\section{1 导管长度在植物内的分布}

尽管某些藤本植物的导管长度可达数十米, 但 植物的大部分导管仍为短导管(Ewers et al., 1990), Ilex verticillata最大导管长度为 $130 \mathrm{~cm}$, 但有 $99.5 \%$ 的导管处在 $0-10 \mathrm{~cm}$ 间, Vaccinium corymbosum最大 导管长度为 130-140 cm, 有 85\% 的导管处于 0$10 \mathrm{~cm}$ 间, 6.5\%的导管处于 $10-20 \mathrm{~cm}$ 间(Zimmermann \& Jeje, 1981), 大部分的导管无法延伸至整个样本。 Venturas 等 (2016) 对 Populus trichocarpa、夏栋 (Quercus robur) 和葡萄进行导管长度分布概率的比 较, 发现其大部分导管长度均在平均导管长度(分 别为 $(1.8 \pm 0.1) 、(10.9 \pm 1.9) 、(11.7 \pm 1.5) \mathrm{cm})$ 之下。 因此, 随样本长度增大, 被切开导管数量急剧下降, 即使被证实拥有较长的导管的植物也是如此。且 Jacobsen等(2012)整合分析发现, 最大导管长度约 为平均导管长度的 5.6 倍。

有研究表明导管长度随枝条的直径、长度或年 龄增大而增大(Zimmermann \& Jeje, 1981; Zimmermann \& Potter, 1982; Ewers \& Fisher, 1989b; Ewers et al., 1990)。对榆树的研究发现, 导管长度随枝条 年龄增大而增长, 纯种Ulmus minor枝条在第5年时 达到稳定值, 而杂交种 $U$. minor $\times U$. pumila在第6 年时达到稳定, $91 \%$ 的最长导管来自最长的枝条, $83 \%$ 的最长导管来自最粗的枝条 (Martín et al., 2013)。对Artemisia tridentata 的根和茎的导管长度做 比较, 发现根的导管长度要明显大于茎(Kolb \& Sperry, 1999)。

\section{2 导管长度在生活型间的分布}

不同生活型的木本植物的平均导管长度具有显 著性差异(Baas et al., 2004; Zhu \& Cao, 2009; Martínez-Cabrera et al., 2011; Gleason et al., 2012; McCulloh et al., 2015; Apgaua et al., 2017), 藤本植 物较灌木及乔木拥有更长的导管, 灌木与乔木的平 均导管长度没有显著差异 (Zimmermann \& Jeje, 1981; Sperry \& Saliendra, 1994)。不区分生活型时, 平均导管长度在不同木材孔隙结构类型上并无差异, 但就乔木而言, 环孔材导管比散孔材更长(Jacobsen et al., 2012), 该结论与前人研究结果一致(Frost,
1930; Cochard et al., 2005, 2010; Choat et al., 2010)。

\section{3 导管长度与导管直径的关系}

导管长度与导管其他结构特征之间有着密切的 关系, 现阶段研究的最多的是其与导管直径的关系 (Zimmermann \& Potter, 1982; Ellmore \& Ewers, 1986; Ewers \& Fisher, 1989b; Kolb \& Sperry, 1999; Hacke \& Jansen, 2009)。不同的研究对于导管直径与导管 长度的关系有不同的结论。从种间水平看有些学者 认为二者相关: Hacke等(2006)通过对28个树种进行 比对, 得出平均导管直径和平均导管长度具有线性 相关性(线性回归曲线的斜率为 $1.48, R^{2}=0.63$ )。对 枫属(Acer)不同种的研究中也发现, 越窄的导管越 短, 导管直径与长度成正相关关系(Lens et al., 2011)。对不同穿孔板类型及导管数量的植物分别研 究发现, 不同类群中, 导管直径均与导管长度成不 同程度的正比关系(Sperry et al., 2007), Jacobsen等 (2012)对 88 个种进行整合分析发现, 种间导管长度 与导管直径仅有弱的相关性 $\left(F_{1,87}=14.09, p<0.001\right.$, $\left.R^{2}=0.14\right)$, 也有些学者认为二者没有关系, 如 Wheeler等(2005)对16个来自不同科属的植物进行 了研究, 并没有发现导管直径与长度之间的相关性; Sperry等(2005)对种子植物研究发现导管长度与导 管直径没有明显的相关性, 而是与导管直径的平方 成正比。对藤本植物的研究也发现, 种间不存在导 管直径与长度的关系, 而在种内研究水平看, 窄导 管普遍较短, 而宽导管则有长有短(Ewers \& Fisher, 1989b; Ewers et al., 1990); Cai等(2010)对杨树种内 无性系研究发现, 导管长度随导管直径增大而增大, 大直径导管趋向于导管长度更长。目前普遍认可的 是种内最大导管直径与最大导管长度成正比(Ewers et al., 1990)。无论种内还是种间, 导管直径在沿导 管方向变异较大, 并不是均一的, 使得导管直径与 导管长度的关系更加多变(Akachuku, 1987; Ewers \& Fisher, 1989b; Brodersen et al., 2011)。

\section{4 导管长度与木质部水力学特性的关系}

\section{1 导管长度与导水率的关系}

与木质部导水率相关的导管特征研究多集中在 导管直径上。根据Hagen-Poiseuille方程, 导管的导 水率与其水力直径 $D_{\mathrm{v}}$ 成正比:

$$
D v=\left(\left(\sum d^{4}\right) / n\right)
$$


其中: $n$ 是所测导管数量, $d$ 是单个导管腔直径(Ewers \& Fisher 1989a; Schreiber et al., 2011)。目前已有大 量研究证明导管直径与导水率相关, 且普遍认为导 管直径与导管长度成正比, 因此导管直径与长度关 系的研究从侧面解释了导管长度与导水率的关系。 当导管较长且弯曲时, 会使导管之间的连接度增大, 使导管网络更加高效协调, 有利于水分的横向运输 (Loepfe et al., 2007; Espino \& Schenk, 2009)。水分在 维管植物木质部导管或篮管(以下简称维管)中运输 时的阻力是由腔阻力与末端壁阻力近似串联而成, 腔阻力是维管内径的函数, 而末端壁的阻力(穿过 穿孔的流阻)则是维管长度的函数。在维管内径相同 时, 导管长度越短, 导管内的端壁数量越少, 水的 流阻越小, 则导水率越大。维管的腔阻力与末端壁 阻力约为 $1: 1$, 但导管越长, 末端阻力越小。可能是 因为导管越长, 拥有纹孔的末端壁重叠区域就越大 (Sperry et al., 2005)。因此，导水率与导管长度的关 系与导管直径、端壁数量及末端壁的重叠区域大小 有关。当导管直径随导管长度增大时, 导水率也随 之增大。就导管而言, 长度越长, 端壁数量越多, 而 末端壁重叠区域也越大, 因此导水率的变化受到二 者综合作用。

Comstock和Sperry (2000)指出导管长度增长时, 单个导管中导管分子数量增多, 就会减少水分在运 输时穿过导管端壁所受到的阻力, 因此是有利于提 高水分运输有效性的进化方向, 但事实上植物体内 的导管并非无限增长的, 这是由于植物受到了安全 性的限制。水分在植物体内的运输是受压力梯度驱 动的, 导管两端存在压力差, 导管末端通常具有更 负的负压，更易受空穴化的威胁。空穴在导管内会 迅速扩展至整个导管, 使其丧失水力功能, 相比之 下短导管的空穴化则对整个茎段的水力功能影响较 小。因此, 导管长度的进化状态是导管水分运输安 全性与有效性的中和效果。

\section{2 导管长度与栓塞脆弱曲线的关系}

\subsection{1 栓塞脆弱曲线的定义}

木质部水势与木质部栓塞的关系可以用“脆弱 曲线” (vulnerability curve, VC) 来表示 (Tyree \& Sperry, 1989), 该曲线是根据木质部导水率损失的 百分数(PLC)与相对应水势绘制而成的曲线, 作为 描述植物水力学特征及水分运输安全性的一个重要
指标，目前已被广泛引用(Maherali et al., 2004)。栓 塞脆弱曲线的建立通常有: 自然干燥法、空气注入 法、压力套法(Cochard et al., 1992)、Cavitron离心机 法(Cochard, 2002; Cochard et al., 2005)和Sperry离心 机技术(Sperry et al., 2010)。以上方法均是将样本置 于不断脱水的环境中诱导并加深栓塞，并测量不同 时期对应的木质部水势与水力功能损失状况, 以此 来判断植物的抗栓塞能力。

\subsection{2 栓塞脆弱曲线测量存在的争议}

离心机法是将枝条放在转子经过改造的离心机 中快速旋转, 通过改变转速不断增大离心力模拟植 株生长过程中的木质部负压来诱导枝条产生栓塞, 从而测定枝条导水率损失百分数(PLC)(Pockman et $a l .$, 1995)。压力套法是将枝条两端切平后, 插入压 力室中, 两端在外, 室中部分树皮划开一部分 (Sperry \& Saliendra, 1994)或完全剥离(Ennajeh et al., 2011), 便于加压时的空气进入以诱导栓塞，同时测 量通过枝条的导水率, 以此来构建脆弱曲线(Cochard et al., 1992; Salleo et al., 1992)。目前对离心机 法与压力套法建立的脆弱曲线的合理性存在着较大 的争议(Christman et al., 2012; Jacobsen \& Pratt, 2012; Sperry et al., 2012; Hacke et al., 2015)。

研究发现: 这两种方法建立栓塞脆弱曲线时, 对具有较长导管的植物，曲线不呈典型的“s”形，而 多为“ “ ”形, 而 “ $\mathrm{r}$ ”形脆弱曲线是否能正确反映出植 物真实的栓塞脆弱性(Cochard et al., 2010)尚不确 定。一种观点认为上述两种方法在建立长导管植物 的栓塞脆弱曲线时, 当导管长度大于样本枝条长度 时, 所得结果大大高估了样本的脆弱性。Cochard等 (2013)在对Choat等(2012)建立的1 200个树种VC曲 线数据库分析后, 对该问题进行了详细的论述, 当 所用样本长度大于最大导管长度时, 由于此时枝条 中开口导管比例小, 用压力套法和离心机法所得结 果接近于真实的 $P_{50}$ (导水率损失 $50 \%$ 时的木质部水 势: 一般用来表示枝条的抗栓塞能力), 而当样本长 度接近或小于最大导管长度时, 所得结果 $\left(P_{50}\right)$ 与实 际值相比要更低, 目前已发表的脆弱曲线中可能有 $20 \%$ 存在该问题。在用压力套法建立脆弱曲线时, 样本的最大导管长度对压力套法所得结果具有关键 性影响。无论是长导管植物还是短导管植物, 均是 在样本长度大于最大导管长度时, 所得脆弱曲线与 
自然干燥法建立的脆弱曲线一致, 枝条短于最大导 管长度时, 结果与自然干燥法与植物在大田中的表 现相比, 显示出了极高的脆弱性 (Ennajeh et al., 2011; Choat et al., 2010)。在对葡萄的研究中, 用不 同方法建立栓塞脆弱曲线, 其 $P_{50}$ 显示出巨大差异 (自然干燥法: $-2.97 \mathrm{MPa}$, 冲洗离心机法: $-0.7 \mathrm{MPa}$ ), 作者认为有可能是由于离心过程中水分会更容易从 开口导管流出, 才造成脆弱性过大的假象(Choat et $a l ., 2010)$ 。也有人认为, 离心机法在使用前要对样 本进行冲洗, 若样本短于最大导管长度, 此时样本 中被切开导管比例较大, 由于开口的导管更容易使 冲洗液中的气泡及其他粒子进入导管, 使枝条的栓 塞脆弱性被高估, 因而造成了“形曲线的假象, 而 短导管植物在测定时样本中被切开导管比例小, 且 短导管纹孔数量少, 膜的直径更小, 气泡与碎屑无 法进入(Choat et al., 2008; Jansen et al., 2009; Limousin et al., 2010; Ennajeh et al., 2011), 因此曲线多 呈“s”形。而压力套法则是枝条中的树汁被空气过饱 和, 且无法被导管滤除, 在枝条末端压力被释放后, 空气在水中的溶解度降低, 并形成气泡, 最终诱发 了空穴化(Wheeler et al., 2013)。

另一种观点则认为长导管植物不会对离心机建 立的栓塞脆弱曲线造成影响。Sperry等(2012)对 Quercus gambelii、复叶槭(Acer negundo)及Sorbus scopulina (平均导管长度分别为17.4、3.1、4.7 cm左 右)不同长度的枝条 $(14 、 27 \mathrm{~cm})$, 用离心机法和自然 干燥法建立脆弱曲线, 结果显示曲线形状及栓塞脆 弱性没有显著差异, 黎明前的Quercus gambelii枝条 用离心机法所测得的VC曲线与自然干燥法所得一 致, 皆证明了导管长度对离心机法建立脆弱曲线没 有影响。对火烧后的Rhamnus ilicifolia次生演替灌木 丛研究表明, 火烧前后, 导管长度没有显著差异, 但脆弱曲线却从火烧前的“s”形变成了火烧后的“r” 形, 这也说明脆弱曲线的形状与导管长度无关 (Jacobsen et al., 2016), 这为“ “"”形曲线真实存在提供 了证据。Barotto等(2016)对桉属3个种(赤桉(Eucalyptus camaldulensis)、E. viminalis、蓝桉 $(E$. globulus)) 的研究发现, 拥有最长导管的种栓塞脆弱 性反而最低 $\left(P_{12}\right.$ 和 $P_{50}$ 更低), 且脆弱曲线为“s”形。

目前针对长导管植物建立脆弱曲线存在的一系 列问题以及“r”形曲线的正确与否, 还需要未来更多 的研究数据, 以及更精准的测量方法来进一步求证。

\section{5 问题和建议}

针对以上与导管长度相关的问题, 特提出以下 几条建议:

1)目前为止，导管长度的测量方法已有了较大 的改进, 染料注入法的灌注物质也经过了数代的变 化, 目前以硅胶注入法最为常用, 硅胶由于本身并 没有颜色, 需要加入苂光剂在紫外线下拍照, 而苂 光剂存在随时间淬灭的现象, 会造成一定的测量误 差(Pan et al., 2015), 因此, 有必要寻找本身显色、黏 滞系数较低, 不易堵塞导管的替代物。导管长度的 测量还多采用计算的方式, 而计算方法的精度还可 以继续提高(Cai \& Tyree, 2014), 在未来的研究中可 以通过更多的实验数据来获得更接近真实导管长度 的计算方法。无论是早期的拍照法, 还是目前广泛 使用的空气注入法和硅胶注入法, 都是将植物离体 测量, 人为将导管剪开, 这有可能对结果造成影响, 近些年兴起的将X射线计算机断层扫描技术应用于 植物解剖学则解决了该问题, 未来可以向动态监测 导管在木质部中的变化过程, 包括导管数量的增多, 小直径导管的增大增长, 以及导管直径与导管长度 的关系随导管生长所发生的变化等方向发展, 在植 物活体上, 动态、连续地观察植物导管的生长过程。

2)导管长度的研究多集中在茎的木质部中, 根 及叶脉中的研究较少(Jacobsen et al., 2012), 但植物 作为土壤-植物-大气连续体中的重要组成环节, 水 分从根部吸收直至通过蒸腾作用回归大气, 需经过 根、茎、叶3个部分的木质部, 因此, 研究植物水力 学特性需从植物整体出发, 了解各个器官的导管分 布状况及各部分的配合作用, 才能更好地掌握植物 的水分运输及利用状况。尽管已有对不同生活型树 种的导管长度的整合分析, 且普遍认为藤本植物拥 有更长的导管，但导管长度分布在种内乃至种间的 变异程度、与植物系统发育学的关系乃至导管形态 是否存在于其他水力学特性协同进化的现象还未可 知, 且目前对导管长度的研究多集中在小范围内的 植物种类, 缺乏对某一类群植物的系统化研究。针 对上述问题, 未来研究可以利用日益完善的数据库 资源来建立不同地域、气候区的导管结构分布图。

3)有关导管直径与导管长度的关系还缺乏具体 定论, 已有研究表明导管直径在整个导管长度方向 上是不均一的(Akachuku，1987; Ewers \& Fisher, 
1989b; Brodersen et al., 2011), 但此现象是具有物种 特殊性, 还是在木本植物中普遍存在? 其生理学意 义还需要进一步探究, 结果或许会对目前大家对二 者关系的普遍认识产生较大的冲击。目前我们所了 解的导管长度与导管其他特性之间的关系, 多集中 在导管直径上, 日后导管长度与连接度、密度等的 关系还需要深入研究, 建立完整的木质部结构模型, 立体化地了解植物导管功能。

4)离心机法与压力套法测得的“r”形曲线的真 实性还存在较大的争议, 论点主要集中在人为切开 的导管造成空气或水的进入上, 若可以在长枝甚至 活体上, 非侵入性的测量植物的栓塞脆弱性, 则会 大大提高测量精度及可信度。近些年以照相机与显 微镜为设备支持的光学测量技术, 为栓塞脆弱性的 测量打开了新的思路(Brodribb et al., 2016; Brodribb et al., 2017; Zhang \& Brodribb, 2017)。光学测量技术 具有操作方便、节约成本、无需离体等明显的优点, 在未来的试验中可能会取代现有的测量方法, 并对 原有的方法或操作造成的错误脆弱曲线进行更正, 可能会改变目前对植物尤其是长导管植物栓塞脆弱 性的认识。

导管的特性对了解植物水力学结构及功能具有 重要的作用, 其中导管长度对评价植物水分运输的 安全性和有效性具有指导性意义。而对植物水力学 特性的了解则会在抗旱品种改良、优良品种选育上 具有重要的作用, 更是为干旱及半干旱地区的植被 恢复及建设提供先决条件。

\section{参考文献}

Akachuku AE (1987). A study of lumen diameter variation along the longitudinal axis of wood vessels in Quercus rubra using cinematography. IAWA Journal, 8, 41-45.

André JP (1998). A study of the vascular organization of bamboos (Poaceae-Bambuseae) using a microcasting method. IAWA Journal, 19, 265-278.

Apgaua DMG, Tng DYP, Cernusak LA, Cheesman AW, Santos RM, Edwards WJ, Laurance SGW (2017). Plant functional groups within a tropical forest exhibit different wood functional anatomy. Functional Ecology, 31, 582-591.

Baas P, Ewers FW, Davis SD, Wheeler EA (2004). Evolution of xylem physiology. In: Hemsley AR, Poole I eds. The Evolution of Plant Physiology. Elsevier Academic Press, London. 273-295.

Barotto AJ, Fernandez ME, Gyenge J, Meyra A, MartinezMeier AM, Monteoliva S (2016). First insights into the functional role of vasicentric tracheids and parenchyma in
Eucalyptus species with solitary vessels: Do they contribute to xylem efficiency or safety? Tree Physiology, 36, 1485-1497.

Brodersen CR, Lee EF, Choat B, Jansen S, Phillips RJ, Shackel KA, McElrone AJ, Matthews MA (2011). Automated analysis of three-dimensional xylem networks using high-resolution computed tomography. New Phytologist, 191, 1168-1179.

Brodersen CR, McElrone AJ, Choat B, Matthews MA, Shackel KA (2010). The dynamics of embolism repair in xylem: In vivo visualizations using high resolution computed tomography. Plant Physiology, 154, 1088-1095.

Brodribb TJ, Bienaimé D, Marmottant P (2016). Revealing catastrophic failure of leaf networks under stress. Proceedings of the National Academy of Science of United States of America, 113, 4865-4869.

Brodribb TJ, Carriqui M, Delzon S, Lucani C (2017). Optical measurement of stem xylem vulnerabilty. Plant Physiology, 174, 2054-2061.

Cai J, Tyree MT (2014). Measuring vessel length in vascular plants: Can we divine the truth? History, theory, methods, and contrasting models. Trees, 28, 643-655.

Cai J, Zhang SX, Tyree MT (2010). A computational algorithm addressing how vessel length might depend on vessel diameter. Plant, Cell \& Environment, 33, 1234-1238.

Choat B, Cobb AR, Jansen S (2008). Structure and function of bordered pits: New discoveries and impacts on wholeplant hydraulic function. New Phytologist, 177, 608-625.

Choat B, Drayton WM, Brodersen C, Matthews MA, Shackel KA, Wada H, Mcelrone AJ (2010). Measurement of vulnerability to water stress-induced cavitation in grapevine: A comparison of four techniques applied to a long-vesseled species. Plant, Cell \& Environment, 33, 1502-1512.

Choat B, Jansen S, Brodribb TJ, Delzon S, Bhaskar R, Bucci SJ, Feild TS, Gleason SM, Hacke UG, Jacobsen AL, Lens F, Maherali H, Martínez-Vilalta J, Mayr S, Mencuccini M, Mitchell PJ, Nardini A, Pittermann J, Pratt RB, Sperry JS, Westoby M, Wright IJ, Zanne AE (2012). Global convergence in the vulnerability of forests to drought. Nature, 491, 752-755.

Christman MA, Sperry JS, Smith DD (2012). Rare pits, large vessels and extreme vulnerability to cavitation in a ring-porous tree species. New Phytologist, 193, 713-720.

Cochard H (2002). A technique for measuring xylem hydraulic conductance under high negative pressures. Plant, Cell \& Environment, 25, 815-819.

Cochard H, Badel E, Herbette S, Delzon S, Choat B, Jansen S (2013). Methods for measuring plant vulnerability to cavitation: A critical review. Experimental Botany, 64, 47794791.

Cochard H, Cruizat P, Tyree MT (1992). Use of positive pressures to establish vulnerability curves: Further support for

www.plant-ecology.com 
the air-seeding hypothesis and implications for pressurevolume analysis. Plant Physiology, 100, 205-209.

Cochard H, Damour G, Bodet C, Tharwat I, Poirier M, Améglio $\mathrm{T}$ (2005). Evaluation of a new centrifuge technique for rapid generation of xylem vulnerability curves. Physiologia Plantarum, 124, 410-418.

Cochard H, Herbette S, Barigah T, Badel E, Ennajeh M, Vilagrosa A (2010). Does sample length influence the shape of xylem embolism vulnerability curves? A test with the Cavitron spinning technique. Plant, Cell \& Environment, 33, 1543-1552.

Cohen S, Bennink J, Tyree MT (2003). Air method measurements of apple vessel length distributions with improved apparatus and theory. Journal of Experimental Botany, 54, 1889-1897.

Comstock JP, Sperry JS (2000). Theoretical considerations of optimal conduit length for water transport in vascular plants. The New Phytologist, 148, 195-218.

Ellmore GS, Ewers FW (1986). Fluid flow in the outermost xylem increment of a ring-porous tree, Ulmus americana. American Journal of Botany, 73, 1771-1774.

Ennajeh M, Simoes F, Khemira H, Cochard H (2011). How reliable is the double-ended pressure sleeve technique for assessing xylem vulnerability to cavitation in woody angiosperms? Physiologia Plantarum, 142, 205-210.

Espino S, Schenk HJ (2009). Hydraulically integrated or modular? Comparing whole-plant-level hydraulic systems between two desert shrub species with different growth forms. New Phytologist, 183, 142-152.

Ewers FW, Fisher JB (1989a). Techniques for measuring vessel lengths and diameters in stems of woody plants. American Journal of Botany, 76, 645-656.

Ewers FW, Fisher JB (1989b). Variation in vessel length and diameter in stems of six tropical and subtropical lianas. American Journal of Botany, 76, 1452-1459.

Ewers FW, Fisher JB, Chiu S (1990). A survey of vessel dimensions in stems of tropical lianas and other growth forms. Oecologia, 84, 544-552.

Frost FH (1930). Specialization in secondary xylem of dicotyledons. I. Origin of vessel. Botanical Gazette, 89, 67-94.

Gleason SM, Butler DW, Zieminska K, Waryszak P, Westoby M (2012). Stem xylem conductivity is key to plant water balance across Australian angiosperm species. Functional Ecology, 26, 343-352.

Gleason SM, Westoby M, Jansen S, Choat B, Hacke HG, Pratt RB, Bhaskar R, Brodribb TJ, Bucci SJ, Cao KF, Chchard H, Delzon S, Domec JC, Fan ZX, Feild TS, Jacobsen AL, Johnson DM, Lens F, Maherali H, Martínez-Vilalta J, Mayr S, McCulloh KA, Mencuccini M, Mitchell PJ, Morris H, Nardini A, Pittermann J, Plavcová L, Schreiber SG, Sperry JS, Wright IJ, Zanne AE (2016). Weak tradeoff between xylem safety and xylem-specific hydraulic effi- ciency across the world's woody plant species. New Phytologist, 209, 123-136.

Hacke UG, Jansen S (2009). Embolism resistance of three boreal conifer species varies with pit structure. New Phytologist, 182, 675-686.

Hacke UG, Sperry JS, Field TS, Sano Y, Skkema EH, Pittermann J (2007). Water transport in vesselless angiosperms: Conducting efficiency and cavitation safety. International Journal of Plant Sciences, 168, 1113-1126.

Hacke UG, Sperry JS, Wheeler JK, Castro L (2006). Scaling of angiosperm xylem structure with safety and efficiency. Tree Physiology, 26, 689-701.

Hacke UG, Venturas MD, MacKinnon ED, Jacobsen AL, Sperry JS, Pratt RB (2015). The standard centrifuge method accurately measures vulnerability curves of longvesselled olive stems. New Phytologist, 205, 116-127.

Jacobsen AL, Pratt RB (2012). No evidence for an open vessel effect in centrifuge-based vulnerability curves of a longvesselled liana (Vitis vinifera). New Phytologist, 194, 982-990.

Jacobsen AL, Pratt RB, Tobin MF, Hacke UG, Ewers FW (2012). A global analysis of xylem vessel length in woody plants. American Journal of Botany, 99, 1583-1591.

Jacobsen AL, Tobin MF, Toschi HS, Percolla MI, Pratt RB (2016). Structural determinants of increased susceptibility to dehydration-induced cavitation in post-fire resprouting chaparral shrubs. Plant, Cell \& Environment, 39, 24732485.

Jansen S, Choat B, Pletsers A (2009). Morphological variation of intervessel pit membranes and implications to xylem function in angiosperms. American Journal of Botany, 96, 409-419.

Kolb KJ, Sperry JS (1999). Transport constraints on water use by the Great Basin shrub, Artemisia tridentata. Plant, Cell \& Environment, 22, 925-936.

Lens F, Sperry JS, Christamn MA, Choat B, Rabaey D, Jansen S (2011). Testing the hypotheses that link wood anatomy to cavitation resistance and hydraulic conductivity in the genus Acer. New Phytologist, 190, 709-723.

Li HF, Tian XH, Ren Y (2005). Research progress in vessel and perforation plate of vascular plants and some considerations for future research. Acta Botanica BorealiOccidentalia Sinica, 25, 419-424. [李红芳, 田先华, 任 毅 (2005). 维管植物导管及其穿孔板的研究进展. 西北 植物学报, 25, 419-424.]

Li R, Jiang ZM, Zhang SX, Cai J (2015). A review of new research progress on the vulnerability of xylem embolism of woody plants. Chinese Journal of Plant Ecology, 39, 838-848. [李荣, 姜在民, 张硕新, 蔡靖 (2015). 木本植 物木质部栓塞脆弱性研究新进展. 植物生态学报, 39, 838-848.]

Limousin J, Longepierre MD, Rambal S (2010). Change in 
hydraulic traits of Mediterranean Quercus ilex subjected to longterm throughfall exclusion. Tree Physiology, 30, 1026-1036.

Loepfe L, Vilalta JM, Ponol J, Mencuccini M (2007). The relevance of xylem network structure for plant hydraulic efficiency and safety. Journal of Theoretical Biology, 247, 788-803.

Maherali H, Pockman WT, Jackson RB (2004). Adaptive variation in the vulnerability of woody plants to xylem cavitation. Ecology, 85, 2184-2199.

Martín JA, Solla A, Ruiz-Villar M, Gil L (2013). Vessel length and conductivity of Ulmus branches: Ontogenetic changes and relation to resistance to Dutch elm disease. Tree, 27, 1239-1248.

Martínez-Cabrera HI, Schenk HJ, Cevallos-Ferriz SR, Jones CS (2011). Integration of vessel traits, wood density, and height in angiosperm shrubs and trees. American Journal of Botany, 98, 915-922.

McCulloh KA, Johnson DM, Petitmermet J, McNellis B, Meinzer FC, Lachenbruch B (2015). A comparison of hydraulic architecture in three similarly sized woody species differing in their maximum potential height. Tree Physiology, 35, 723-731.

Pan RH, Geng J, Cai J, Tyree MT (2015). A comparison of two methods for measuring vessel length in woody plants. Plant, Cell \& Environment, 38, 2519-2526.

Pockman WT, Sperry JS, O’Leary JW (1995). Sustained and significant negative water pressure in xylem. Nature, 378, 715-716.

Salleo S, Hinckley TM, Kikuta SB, Lo Gullo MA, Weilgony P, Yoon TM, Richter H (1992). A method for inducing xylem emboli in situ: Experiments with a field-grown tree. Plant, Cell \& Environment, 15, 491-497.

Schreiber SG, Hacke UG, Hamann A (2011). Genetic variation of hydraulic and wood anatomical traits in hybrid poplar and trembling aspen. New Phytologist, 190, 150-160.

Skene DS, Balodis V (1968). A study of vessel length in Eucalyptus oblique L'Hérit. Journal of Experimental Botany, 19, 825-830.

Sperry JS, Christman MA, Torres-Ruiz JM, Taneda H, Smith DD (2012). Vulnerability curves by centrifugation: Is there an open vessel artefact, and are ' $r$ ' shaped curves necessarily invalid? Plant, Cell \& Environment, 35, 601-610.

Sperry JS, Donnelly JR, Tyree MT (2010). A method for measuring hydraulic conductivity and embolism in xylem. Plant, Cell \& Environment, 11, 35-40.

Sperry JS, Hacke UG, Field TS, Sano Y, Sikkema EH (2007). Hydraulic consequences of vessel evolution in angiosperms. International Journal of Plant Sciences, 168, 1127-1139.

www.plant-ecology.com
Sperry JS, Hacke UG, Wheeler JK (2005). Comparative analysis of end wall resistivity in xylem conduits. Plant, Cell \& Environment, 28, 456-465.

Sperry JS, Saliendra NZ (1994). Intra- and inter-plant variation in xylem cavitation in Betula occidentalis. Plant, Cell \& Environment, 17, 1233-1241.

Sperry JS, Tyree MT (1988). Mechanism of water stress-induced xylem embolism. Plant Physiology, 88, 581- 587.

Tyree MT (1993). Theory of vessel-length determination: The problem of nonrandom vessel ends. American Journal of Botany, 71, 297-302.

Tyree MT, Dixon MA (1986). Water stress induced cavitation and embolism in some woody plants. Physiologia Plantarum, 66, 397-405.

Tyree MT, Sperry JS (1989). Vulnerability of xylem to cavitation and embolism. Annual Review of Plant Physiology and Plant Molecular Biology, 40, 19-38.

Tyree MT, Zimmermann MH (2002). Xylem Structure and the Ascent of Sap. 2nd edn. Springer, Berlin.

Venturas MD, Rodriguez-Zaccaro FD, Percolla MI, Crous CJ, Jacobsen AL, Pratt RB (2016). Single vessel air injection estimites of xylem resistance to cavitation are affected by vessel network characteristics and sample length. Tree physiology, 36, 1247-1259.

Wheeler JK, Huggett BA, Tofte AN, Rockwell FE, Holbrook NM (2013). Cutting xylem under tension or supersaturated with gas can generate PLC and the appearance of rapid recovery from embolism. Plant, Cell \& Environment, 36, 1938-1949.

Wheeler JK, Sperry JS, Hacke UG, Hoang N (2005). Intervessel pitting and cavitation in woody Rosaceae and other vesselled plants: A basis for a safety versus efficiency trade-off in xylem transport. Plant, Cell \& Environment, 28, 800-812.

Zhang FP, Brodribb TJ (2017). Are flowers vulnerable to xylem cavitation during drought? Proceedings Biological Science, 284, 20162642. DOI: 10.1098/rspb.2016.2642.

Zhu SD, Cao KF (2009). Hydraulic properties and photosynthetic rates in co-occurring lianas and trees in a seasonal tropical rainforest in southwestern China. Plant Ecology, 204, 295-304.

Zimmermann MH, Jeje AA (1981). Vessel-length distribution in stems of some American woody plants. American Journal of Botany, 59, 1882-1892.

Zimmermann MH, Potter D (1982). Vessel-length distribution in branches, stem and roots of Acer rubrum L. International Association of Wood Anatomists Bulletin, 3, 103-109. 